\title{
O PERCURSO PROFISSIONAL DE MANFREDO PERDIGÃO DO CARMO E A GEOMETRIA DIFERENCIAL NO BRASIL
}

\section{THE PROFESSIONAL CAREER OF MANFREDO PERDIGÃO DO CARMO AND THE DIFFERENTIAL GEOMETRY IN BRAZIL}

\author{
Antonio José Melo de Queiroz ${ }^{1}$ \\ Universidade Estadual do Ceará
}

\section{Resumo}

Este trabalho elaborou uma visão panorâmica sobre o início da carreira do professor e pesquisador brasileiro Manfredo Perdigão do Carmo (1928 - 2018). Será discutida de forma breve sua formação em Engenharia Civil, a pós-graduação em Geometria Diferencial e suas atividades iniciais de pesquisa e ensino. No texto será abordada a produção científica deste eminente geômetra, uma breve exposição dos primeiros artigos científicos, formação de parcerias de pesquisa, orientação de diversos estudantes em nível de doutorado e o constante esforço para colaborar com a produção de conhecimento matemático no Brasil. Diversos artigos publicados pelo professor Manfredo foram elaborados em parcerias com matemáticos brasileiros ou estrangeiros. Essas colaborações se ampliavam à medida que Manfredo do Carmo orientava vários estudantes de pós-graduação em Matemática no Brasil, principalmente, no Instituto de Matemática Pura e Aplicada (IMPA), além disso, estas e outras atividades de ensino colaboraram para a formação de uma fecunda escola de Geometria Diferencial no país. Manfredo do Carmo também escreveu uma série de livros, a maioria deles dedicados à Geometria Diferencial, algumas destas obras são referências de prestígio nacional e internacional. Além das atividades de ensino e pesquisa, o matemático esteve envolvido com ações de gerência institucional, foi presidente da Sociedade Brasileira de Matemática (SBM) e membro da Academia Brasileira de Ciências (ABC). Diante do exposto acima, este estudo teve como objetivos compreender e analisar o início do percurso profissional do professor Manfredo Perdigão do Carmo bem como sua importância para a formação da área de estudos da Geometria Diferencial no Brasil. Será dado destaque que ele foi um dos precursores deste tema em nosso país, pois antes de sua atuação existiam apenas alguns trabalhos esparsos realizados por matemáticos pioneiros entre o fim do século XIX e início do século XX. A metodologia adotada nesta pesquisa é do tipo exploratória, permitindo uma maior aproximação e compreensão do objeto de estudo, baseada em análises bibliográficas e, principalmente, análises documentais, com destaque para a investigação dos artigos científicos, além da observação de documentos em que constam suas atividades de orientação de teses de doutorado e entrevistas concedidas por Manfredo do Carmo, em que trata de diversos pontos de sua carreira profissional. Alguns resultados observados na pesquisa são a trajetória acadêmica exitosa e rica em detalhes, bem como o árduo trabalho do professor Manfredo para criar e consolidar uma escola de Geometria Diferencial no Brasil,

1 antonio.queiroz@uece.br

Número Especial - IV Seminário Cearense de História da Matemática

Boletim Cearense de Educação e História da Matemática - Volume 07, Número 20, 266 - 276 (2020)

DOI: 10.30938/bocehm.v7i20.2819 
mantendo um alto nível de produção científica. A conclusão obtida ressalta a importância das atividades de pesquisa e ensino de Manfredo do Carmo, bem como suas parcerias na realização das atividades de pesquisa.

Palavras-chave: Manfredo do Carmo; Geometria; História da Matemática.

\section{Abstract}

This paper elaborated a panoramic view about the beginning of the career of Brazilian teacher and researcher Manfredo Perdigão do Carmo (1928 - 2018). It will be briefly discussed his background in Civil Engineering, the postgraduate degree in Differential Geometry and his initial research and teaching activities. The text will address the scientific production of this eminent geometer, a brief exposition of the first scientific articles, formation of research partnerships, orientation of several $\mathrm{PhD}$ students and the constant effort to collaborate with the production of mathematical knowledge in Brazil. Several articles published by Professor Manfredo were written in partnership with Brazilian or foreign mathematicians. These collaborations were expanding as Manfredo do Carmo mentored several postgraduate students in Mathematics in Brazil, mainly at the Institute of Pure and Applied Mathematics (IMPA). In addition, these and other teaching activities contributed to the formation of a fertile school of Differential Geometry in the country. Manfredo do Carmo has also written a series of books, most of them devoted to Differential Geometry, some of which are references of national and international prestige. In addition to teaching and research activities, the mathematician was involved in institutional management activities, was president of the Brazilian Society of Mathematics (SBM) and a member of the Brazilian Academy of Sciences $(\mathrm{ABC})$. Given the above, this study aimed to understand and analyze the beginning of the professional career of Professor Manfredo Perdigão do Carmo as well as its importance for the formation of the field of studies of Differential Geometry in Brazil. It will be noted that he was one of the forerunners of this theme in our country, because before his performance there were only a few sparse works by pioneering mathematicians between the late nineteenth and early twentieth century. The methodology adopted in this research is exploratory, allowing a closer approach and understanding of the object of study, based on bibliographical analysis and, mainly, documentary analysis, with emphasis on the investigation of scientific articles, in addition to the observation of documents containing their $\mathrm{PhD}$ thesis orientation activities and interviews given by Manfredo do Carmo, in which deals various points of your professional career. Some results observed in the research are the successful and detailed academic trajectory, as well as the hard work of Professor Manfredo to create and consolidate a school of Differential Geometry in Brazil, maintaining a high level of scientific production. The conclusion obtained underscores the importance of Manfredo do Carmo's research and teaching activities, as well as his partnerships in conducting research activities.

Keywords: Manfredo do Carmo; Geometry; Mathematics History.

\section{Introdução}

O sucesso recente da Matemática brasileira esconde a sua origem tardia e difícil.

Atualmente, o Brasil encontra-se no grupo 5 da International Mathematical Union 
(IMU), ou seja, o país está no conjunto de países que mais produzem Matemática de qualidade no mundo. Porém, ao longo da história da ciência nacional, percebe-se que a Matemática superior, com raras excessões, foi ensinada somente em escolas de engenharia até a década de 1930. Assim, não havia uma formação oficial de cientistas matemáticos até essa data, isto não significa a ausência de estudos nesta área, existiram alguns nomes fortes e autodidatas que conseguiram realizar trabalhos de pesquisa importantes em algumas áreas da Matemática, alguns deles foram, Joaquim Gomes de Sousa, Theodoro Augusto Ramos, Otto de Alencar Silva, Lélio Gama, dentre outros, conhecidos como pioneiros da Matemática brasileira.

Manfredo Perdigão do Carmo, um dos matemáticos mais importantes do Brasil, iniciou sua carreira em um segundo momento da Matemática nacional, pois quando concluiu sua graduação em Engenharia Cívil em 1951 e posteriormente quando decidiu concentrar esforços à docência e a iniciação científica em Matemática, já existiam algumas faculdades dedicadas ao ensino e pesquisa em ciência básica, situadas no eixo Rio de Janeiro - São Paulo. Assim, apesar da irrelevante ou inexistente divulgação destas atividades, já existia a semente da produção científica nacional germinando em solo brasileiro.

Em conformidade com o apresentado acima, traz-se nesta pesquisa o objetivo de compreender e analisar o início da trajetória profissional do professor Manfredo Perdigão do Carmo, entre as décadas de 1960 e 1970, bem como, apresentar a sua importância para a formação da área de Geometria Diferencial, no Brasil, visto que até o início de suas atividades de pesquisador, existiam poucos trabalhos nacionais neste campo de estudo.

$\mathrm{O}$ percurso metodológico trilhado neste trabalho foi à pesquisa documental e bibliográfica com um caráter exploratório e qualitativo, tendo em vista a análise de algumas entrevistas do professor Manfredo do Carmo, documentadas em vídeos e textos, bem como o estudo de material bibliográfico sobre História da Matemática no Brasil e leitura de alguns trabalhos de pesquisa e livros publicados pelo professor Manfredo.

Os principais resultados apresentados são a relevância da produção científica de Manfredo do Carmo, observando que a maior parte de suas investigações em Geometria Diferencial foram realizadas em parceria com importantes geômetras. É destacado 
também as suas atividades de ensino sob a forma de orientação de vários estudantes brasileiros no IMPA que, posteriormente, se deslocaram para diversas universidades e formaram novos núcleos na área relatada acima.

\section{Breve biografia}

Manfredo Perdigão do Carmo nasceu em Maceió, AL, em 1928, onde também fez sua educação básica, na época, ensino primário e secundário. Um fato interessante deste período é que ele foi aluno de Benedito de Morais, assim como Elon Lages Lima. Manfredo se refere ao memorável professor com muito respeito e carinho relatando que "[...] era uma pessoa diferenciada, que fazia aquilo com gosto, com amor, com o maior entusiasmo."(CARMO, 2003, p. 199). Percebemos que Benedito de Morais era um mestre que cativava e marcava seus estudantes, pois Manfredo com mais de 70 anos de idade ainda lembrava de suas aulas no ensino básico.

Em 1947, Manfredo do Carmo iniciou o curso de Engenharia Cívil na Escola de Engenharia de Recife, nessa instituição sofreu influência de outro insigne professor, Luiz Freire. De acordo com Silva (2013), a visão de futuro de Luiz Freire, associada às parcerias com outros matemáticos, fez a Universidade Federal de Pernambuco (UFPE) ser um importante centro de produção e divulgação da Matemática. Manfredo destaca o estímulo, promovido por Luiz Freire, aos estudantes para seguirem carreira científica (CARMO, 1994).

Já graduado, em 1951, Manfredo retorna a Maceió e realiza alguns trabalhos em engenharia, porém, Luiz Freire o indicou, em 1954, para uma posição no Instituto Tecnológico da Aeronaútica e, no mesmo ano, Manfredo retorna para Recife com o objetivo de ser professor no recém criado Instituto de Física e Matemática da Universidade de Recife (atual UFPE), iniciando sua frutífera carreira docente e a intenção em estudos profundos da Matemática.

O período entre 1955 e 1960 foi dedicado à preparação matemática e aprendizagem de assuntos que ainda não tinha conhecimento consolidado. Manfredo do Carmo relata que entre 1955 e 1956 dedicou-se ao preenchimento de falhas em sua formação e, como era admirador de Física, desenvolveu, pela primeira vez, o interesse em Geometria Diferencial. O ano de 1957 foi particularmente importante por sua participação no $1^{\circ}$ Colóquio Brasileiro de Matemática (CBM). Carmo (1994) narra que, 
neste evento, percebeu pela primeira vez a existência de pessoas, no Brasil, com interesses semelhantes aos seus, conheceu alguns matemáticos como Chaim Samuel Honig, Alexandre Rodrigues e soube que seu amigo de infância, Elon Lages, estava cursando doutorado em Topologia, nos Estados Unidos.

A partir do colóquio, o professor Manfredo iniciou e manteve uma correspondência valiosa com Elon, que indicou uma série de livros e notas de aulas para aprofundamento nos estudos de matemática e, principalmente, de Geometria Diferencial. Em 1958, os dois se reencontraram em Fortaleza, Elon já era doutor e Manfredo havia sido convidado para ministrar um curso de Geometria, que foi o germe para a escrita de seu livro mais famoso, Differential Geometry of Curves and Surfaces. Após algumas reflexões com o amigo, o professor resolveu passar um período de estágio no IMPA, entre 1959 e 1960, lá, sob orientação de Elon Lages, aprofundou estudos em Geometria Diferencial e conforme relatou "pela primeira vez na vida eu estava em contato com a matemática nascente.” (CARMO, 2003, p. 203).

Em outubro de 1960, Manfredo do Carmo chegou à Universidade de Berkeley para cursar doutorado em Matemática, na área de Geometria Diferencial e sob orientação de um dos maiores geômetras da época, Shiing-Shen Chern. Manfredo foi indicado por cartas de referências de Elon Lages, Leopoldo Nachbin e Maurício Peixoto. Vale destacar que seu objetivo era trabalhar com um grande matemático, assim relatou, “[...] tomei a decisão de me dedicar inteiramente à Matemática. Decorria dai que eu devia fazer o Doutorado em uma boa Universidade. Como eu estava interessado em Geometria Diferencial, era natural procurar estudar com S.S.Chern.” (CARMO, 1994, P. 3).

Após a elaboração de uma prestigiada tese e conclusão do doutorado, o professor Manfredo retornou ao Brasil e entre 1963 e 1967 colaborou com várias universidades brasileiras e foi, nas palavras de Clóvis Pereira da Silva, “[...] quem mais contribuiu, ao lado de Alexandre Augusto M. Rodrigues, para o desenvolvimento e consolidação da pesquisa em Geometria Diferencial no Brasil.” (SILVA, 2009, p. 49). No retorno, esteve na sua instituição de origem, Universidade de Recife. Em 1965, trabalhou na Universidade de Brasília, onde iniciou as atividades de pesquisa com Elon Lages. A partir de 1966, ingressou como pesquisador do IMPA, instituição em que trabalhou até a sua morte, em 2018. Ainda em 1966, participou da formação da pós-graduação em 
Matemática na Universidade Federal do Ceará (UFC), onde ministrou diversos cursos, após esse período retornou aos Estados Unidos com o auxílio de uma renomada bolsa Guggenheim.

Durante a estadia em solo americano, Manfredo do Carmo realizou importantes trabalhos de pesquisa, que serão discutidos posteriormente, e, além disso, firmou importantes parcerias. De volta ao Brasil, em 1969, fixou-se no IMPA, onde iniciou um trabalho de formação de jovens talentosos e consolidação da pesquisa em Geometria Diferencial no Brasil.

Manfredo também esteve ligado a outras funções importantes para a ciência nacional, tendo sido presidente da SBM entre 1971 e 1973 e membro da ABC, a partir de 1970. Observamos que o professor foi ativo e acreditou na possibilidade da formação de uma comunidade matemática nacional. Será apresentado nas próximas seções que Manfredo elaborou profundos trabalhos de pesquisa em colaboração com grandes matemáticos, escreveu diversos livros e, além disso, formou excelentes geômetras.

\section{Primeiros artigos científicos e livros}

O primeiro artigo científico de Manfredo do Carmo foi resultado de sua tese de doutorado, publicado no Annals of Mathematics, periódico americano e um dos mais prestigiados do mundo. O trabalho, nas palavras de Marques (2018), estudava as relações entre a topologia e a curvatura de alguns objetos geométricos e representou um ótimo início de carreira para o matemático.

Durante a segunda estadia nos Estados Unidos, entre 1967 e 1969, o professor Manfredo produziu pesquisas em parcerias com Elon Lages, Frank Warner, Nolan Wallach, Shiing-Shen Chern, Shoshichi Kobayashi e outros. Com Elon Lages, publicou um artigo no Archiv der Mathematik em que obtiveram “[...] uma condição para que uma variedade compacta $\mathrm{M}^{\mathrm{n}}$ do espaço euclideano $\mathrm{R}^{\mathrm{n}+\mathrm{p}}$ estivesse contida em $\mathrm{R}^{\mathrm{n}+1}$.”(CARMO, 1994, p. 8). É importante destacar que este texto é citado em quase todas as "revisões bibliográficas" escritas sobre o assunto (CARMO, 1994).

Ainda durante este período, Manfredo do Carmo escreveu em colaboração com S. S. Chern e S. Kobayashi seu trabalho de pesquisa mais citado pela comunidade matemática, Minimal submanifolds of a sphere with second fundamental forms of constant length, publicado no periódico Functional Analysis and related fields. A partir 
deste período, o professor Manfredo desenvolveu o interesse pela subárea de superfícies mínimas, assunto em que contribuiu até o fim de sua carreira.

Após o breve relato dos primeiros artigos científicos publicados pelo professor Manfredo, deve-se destacar alguns fatos importantes, tais como, a realização de colaborações, a importância de suas contribuições para a Matemática, e que seus escritos foram, com raras exceções, os primeiros publicados por um brasileiro na área de Geometria Diferencial.

Corroborando com o que foi citado no parágrafo anterior, Tenenblat (2018) afirma que Manfredo do Carmo produziu mais de 80 trabalhos científicos em cooperação com 32 matemáticos e atuou em diversos temas de sua área tais como, as relações entre Topologia e curvatura, convexidade e rigidez, imersões conformes, subvariedades mínimas e outros.

Além da pesquisa em Geometria Diferencial, Manfredo valorizava a produção de livros de Matemática em língua materna. Tenenblat (2018), lembra que algumas de suas obras eram "testadas" nas disciplinas que ministrava e Barbosa (2018) reforça que, durante o período em que Manfredo lecionou na UFC, o livro de Geometria Diferencial não continha exercícios e estes eram elaborados ao longo do curso para a inclusão no texto. Além disso, o professor utilizava a opinião da turma como "medida" para avaliação de cada lista de problemas.

O livro citado no parágrafo anterior pelo professor Lucas Barbosa foi o primeiro texto didático produzido por Manfredo do Carmo, cujo título é Geometria Diferencial Local, e representava "[...] um esquema para a parte básica do curso de Geometria Diferencial.” (CARMO, 1963, p. 3). Esta obra é composta de cinco capítulos que tratam de curvas, superfícies regulares e algumas propriedades, como a primeira forma fundamental, superfícies imersas e a segunda forma fundamental, geometria intríseca das superfícies e o teorema fundamental para estes objetos matemáticos.

Este livro foi publicado em 1963 pelo Instituto de Física e Matemática da Universidade do Recife, na coleção Textos de Matemática, sob a coordenação de Alfredo Pereira Gomes, além disso, foi utilizado como notas de aula para um curso ministrado por Manfredo no $4^{\circ}$ Colóquio Brasileiro de Matemática. Outra obra didática do professor Manfredo, muito famosa, é intitulada Differential Geometry of Curves and 
Surfaces, publicada em 1976 pela editora Prentice Hall, nos Estados Unidos, posteriormente traduzida para o português e publicada pela SBM.

Tenenblat (2018) lembra que este livro nasceu de uma obra preliminar publicada pelo IMPA, Elementos de Geometria Diferencial, em 1971, e Manfredo do Carmo cita a influência do matemático Blaine Lawson para que o referido texto fosse publicado em solo americano, pois ressalta que, em uma temporada no Rio de Janeiro, Lawson estudou o livro, o estimulou à tradução para o inglês e iniciou tal tarefa, que foi concluida por Leny Cavalcante, esposa de Manfredo. O professor ainda destaca sua surpresa ao saber que o texto já teria vendido mais de 30 mil exemplares (CARMO, 2003).

Esses dois textos de Manfredo do Carmo foram citados por pertencerem ao período inicial de sua carreira e mesmo assim, um destes tornou-se referência em diversas universidades nacionais e estrangeiras. Através desta observação é possível perceber o empenho e o talento do professor Manfredo para a escrita de Matemática, bem como o interesse na consolidação bibliográfica nacional, tendo como consequência o estímulo ao estudo e pesquisa em Matemática no Brasil. Na próxima seção será discutido brevemente algumas atividades de ensino realizadas pelo matemático, que tem uma vasta descendência acadêmica.

\section{Atividades de ensino}

Manfredo Perdigão do Carmo envolveu-se ativamente em diversas atividades de ensino ao longo de sua carreira, ele relatou que "[...] havia essa preocupação de criar uma matemática nacional, era preciso ter alunos que fizessem teses, trabalhos de pesquisa aqui no Brasil, com problemas tirados daqui.” (CARMO, 2003, p. 217). Em 1969, no retorno às suas atividades no IMPA, Manfredo assumiu a direção de ensino no instituto, cargo que lidava com a estruturação e manutenção das atividades de pósgraduação. Além disso, ministrou vários cursos como, Geometria Diferencial Clássica, Formas Diferenciais e Geometria Riemanniana, organizou seminários avançados e orientou alunos de doutorado (CARMO, 1994).

A orientação de alunos na elaboração de suas teses foi uma das principais atividades contribuintes para a formação da escola de Geometria Diferencial no Brasil, uma vez que, boa parte desses jovens doutores retornaram aos seus estados de origem 
ou assumiram postos de trabalho fora do eixo Rio de Janeiro - São Paulo e estimularam a criação de novos grupos nesta área de pesquisa.

Neste sentido, Tenenblat (2018) relata que Manfredo foi orientador de 27 teses de doutorado. Estes novos doutores continuaram suas pesquisas e, por sua vez, passaram a orientar novos alunos, sendo assim, muitos geômetras brasileiros estão ligados, direta ou indiretamente, a Manfredo Perdigão do Carmo.

Será citado, em ordem cronológica, os orientandos de Manfredo que realizaram o doutoramento na década de 1970: Keti Tenenblat (1972), Rubens Leão de Andrade (1973), Edmilson de Vasconcelos Pontes (1974), José de Anchieta Delgado (1977), Antonio Carlos Asperti (1977), Ivan Azevedo Tribuzy (1978), Renato de Azevedo Tribuzy (1978) e Luquésio Petrola de Melo Jorge (1978) (SILVA; AZEVEDO, S/d). Estes foram os primeiros estudantes que realizaram trabalhos de tese sob supervisão de Manfredo do Carmo.

Importante destacar que as pessoas citadas acima passaram a realizar suas atividades de ensino e pesquisa em diversos estados do país como, Distrito Federal, Ceará, Rio Grande do Norte, São Paulo e Amazonas. Esse fato evidencia a importância das atividades realizadas por Manfredo do Carmo para a formação do campo de pesquisa e ensino de Geometria Diferencial no país. Na próxima seção, será abordado algumas breves considerações finais desta pesquisa.

\section{Considerações finais}

Ao concluir esta pesquisa, pode-se ressaltar alguns pontos relevantes no início da carreira do professor Manfredo do Carmo. Primeiro, sua produção científica expressiva em quantidade e qualidade, dado que, sua primeira publicação já ocorreu no Annals of Mathematics, uma das revistas de Matemática mais pretigiada do mundo. Além disso, podemos destacar a grande quantidade de parceiros de pesquisa, Manfredo valorizava a produção conjunta e a praticou até o fim de sua vida.

O segundo ponto a ser notado é a preocupação com a formação de uma bibliografia em língua portuguesa. Manfredo do Carmo escreveu, desde o seu retorno ao Brasil, depois do doutorado, várias notas de aulas dos cursos que ministrava. Alguns destes textos se tornaram livros célebres e referências em Geometria Diferencial, no Brasil e no exterior. 
O terceiro aspecto observado foi à formação de profissionais para atuarem em ensino e pesquisa da Geometria Diferencial no Brasil. Manfredo orientou vários estudantes de mestrado e doutorado, foram citados os primeiros orientandos de doutorado, em ordem cronológica, observando que estas pessoas passaram a desenvolver diversas atividades na área e, em várias regiões do país, colaborando de forma decisiva para a formação da comunidade de Geometria Diferencial.

A partir destes pontos destacados, foi ressaltado o desenvolvimento do objetivo da pesquisa, uma vez que, foi discutida a importância científica dos artigos e livros de Manfredo do Carmo, bem como as atividades de orientação de teses de doutorado, realizados no IMPA. É importante lembrar que foram analisadas apenas algumas atividades iniciais da carreira do professor Manfredo, entre as décadas de 1960 e 1970. Seu percurso profissional completo é muito amplo e rico em detalhes, espera-se que seja tema de outras pesquisas. O autor agradece ao professor Diego da Silva Pinheiro, doutorando em Geometria Diferencial da UFC, por suas valiosas contribuições.

\section{Referências}

BARBOSA, J. L. M et al. Mesa Redonda Recordando Manfredo. In: JORNADA MANFREDO DO CARMO, 2018, Rio de Janeiro. Disponível em: https://www.youtube.com/watch?v=pyiX_P3pD5c\&t=138s. Acesso em: 25 novembro 2019.

CARMO, M. P. Manfredo Perdigão do Carmo. In: PALIS, J.; CAMACHO, C.; LIMA, E. L. (orgs.) IMPA 50 anos. Rio de Janeiro: IMPA, 2003. p. 199-222.

CARMO, M. P. Manfredo Perdigão do Carmo. [Entrevista cedida a] Pedro Mendes. Matemática Universitária, Rio de Janeiro, n. 16, p. 1-18, jul. 1994.

CARMO, M. P. Geometria Diferencial Local. Recife: Instituto de Física e Matemática da Universidade de Recife, 1963.

MARQUES, F. C. S. C. A Matemática de Manfredo Perdigão do Carmo. In: JORNADA MANFREDO DO CARMO, 2018, Rio de Janeiro. Disponível em: https://www.youtube.com/watch?v=4vb5jHfDuKk\&t=116s. Acesso em: 14 novembro 2019.

SILVA, C. P. Início e Consolidação da Pesquisa em Matemática do Brasil. 2 ed. Rio de Janeiro: Editora Ciência Moderna Ltda, 2013.

SILVA, C. P. Aspectos Históricos do Desenvolvimento da Pesquisa Matemática no Brasil. São Paulo: Editora Livraria da Física, 2009. 
SILVA, C. P.; AZEVEDO, A. Mestrados e Doutorados em Matemática Obtidos no Brasil a Partir de 1942. Sociedade Brasileira de História da Matemática, Rio Claro, S/d. Disponível em: www.sbhmat.com.br. Acesso em: 10 setembro 2017.

TENENBLAT, Keti. O Legado de Manfredo Perdigão do Carmo. In: JORNADA MANFREDO DO CARMO, 2018, Rio de Janeiro. Disponível em: https://www.you tube.com/watch?v=umMK2Wa8n-E\&t=473s. Acesso em: 20 novembro 2019. 